Research Paper

\title{
A novel blood tool of cancer prognosis in esophageal squamous cell carcinoma: the Fibrinogen/Albumin Ratio
}

\author{
Zihui Tan ${ }^{1,2,3 *}$, Man Zhang4*, Qiang Han5*, Jing Wen ${ }^{1,2,3}$, Kongjia Luo ${ }^{1,2,3}$, Peng Lin ${ }^{1,2,3}$, Lanjun Zhang ${ }^{1,2,3}$, \\ Hong Yang ${ }^{1,2,3}$, Jianhua $\mathrm{Fu}^{1,2,3 凶}$ \\ 1. State Key Laboratory of Oncology in South China, Collaborative Innovation Center for Cancer Medicine. \\ 2. Department of Thoracic Surgery, Sun Yat-sen University Cancer Center, 651 Dongfeng Road East, Guangzhou, China. \\ 3. Guangdong Esophageal Cancer Institute, Guangzhou, China. \\ 4. Department of Ultrasound, The third affiliated hospital, SunYat-senUniversity, 600 Tianhe Road, Guangzhou, China. \\ 5. Department of Thoracic Surgery, The fifth affiliated hospital, SunYat-senUniversity, Zhuhai, China. \\ * These authors contributed equally to this work \\ $\triangle$ Corresponding author: Jianhua Fu, Department of Thoracic Surgery, Sun Yat-sen University Cancer Center, 651 Dongfeng Road East, Guangzhou, China, \\ Post Code: 510060. Email: jianhua_fu@163.com; Mobile phone: +8613902401851; Fax: +862087343628 \\ (c) Ivyspring International Publisher. This is an open access article distributed under the terms of the Creative Commons Attribution (CC BY-NC) license \\ (https://creativecommons.org/licenses/by-nc/4.0/). See http://ivyspring.com/terms for full terms and conditions.
}

Received: 2016.06.15; Accepted: 2016.12.22; Published: 2017.04.08

\begin{abstract}
Background: Coagulation and nutrition play important roles in cancer progression. We aim to investigate the impact of the fibrinogen/albumin ratio(FAR) in esophageal squamous cell carcinoma (ESCC) patients.

Methods: We retrospectively analyzed 1135 patients with radical esophagectomy for ESCC from January 2008 to December 2010 in our center. X-tile software was used to determine the optimal cutoff levels for these biomarkers.

Results: The optimal cutoff value was 0.08 for the FAR by the X-tile software. The FAR was statistically significantly associated with age $(p=0.003)$, $\operatorname{sex}(p=0.030)$, tumor length $(p=0.043), \mathrm{PT}$ status $(p<0.001)$ and $\mathrm{pN}$ status $(p<0.001)$. Pearson's correlation indicated that the FAR were positively associated with the serum C-reactive protein (CRP) $(r=0.583, p<0.001)$, and the NLR ( $r=0.316, p<0.001$ ). Multivariate analysis indicated that age, tumor grade, $\mathrm{pT}$ status, $\mathrm{pN}$ status and preoperative FAR were independent prognostic factors in patients with ESCC.

Conclusions: Preoperative FAR was an independent prognostic factor in ESCC patients. Lower FAR may improve OS of ESCC patients.
\end{abstract}

Key words: esophageal squamous cell carcinoma, blood tool, fibrinogen, albumin

\section{Background}

Esophageal cancer is one of the most common malignancy worldwide[1]. In China, the major type of esophageal cancer is esophageal squamous cell carcinoma (ESCC) [2]. Growing evidence indicates that neoadjuvant chemoradiotherapy may be more appropriate for locally advanced esophageal cancer while surgery is the main treatment strategy for early stage disease[3,4]. Thus, accurate preoperative staging helps the clinician to determine the treatment strategy. However, preoperative staging tools such as tumor markers, computed tomography (CT), endoscopic ultrasound (EUS) and positron emission tomography (PET) may be inaccurate. Identifying a simple and convenient indicator to stratify subgroups of patients is needed.

It has been reported that coagulation was associated with a more malignant disease [5,6]. Fibrinogen, a glycoprotein synthesized by liver epithelium, plays an essential role in the coagulation cascade, converting to fibrin monomers and ultimately forming the fibrin polymers[7]. Previous studies indicated that elevated preoperative 
fibrinogen level might induce higher malignance progression and poor prognosis[5,6]. However, few studies have investigated the predictive impact of preoperative fibrinogen levels in ESCC patients. In addition, it has been demonstrated that malnutrition is a predictor in various cancers[8], and albumin is regarded as a nutritional indicator. Hypoalbuminemia was found to be associated with poor prognosis in various cancers, including lung, gastric, and colon cancers[8].

Therefore we established a novel prognostic maker, fibrinogen/albumin ratio (FAR), investigating its prognostic impact in ESCC patients.

\section{Methods}

\section{Patients}

We reviewed the data of ESCC patients with radical esophagectomy at the Sun Yat-sen University Cancer Center between January 2008 to December 2010. We excluded patients received neoadjuvant or adjuvant therapy and with $\mathrm{R} 1 / \mathrm{R} 2$ resection. We also excluded patients who received anticoagulant treatment or albumin transfusions before treatment. Preoperative assessment included routine blood examination, pulmonary function test, electrocardiography, esophageal radiography, CT scans of the chest and upper abdomen, cervical ultrasonography, and an upper gastrointestinal endoscopy or EUS with biopsy before treatment. All patients were performed by radical esophagectomy and two-field or three-field lymphadenectomy. Three-field lymphadenectomy was performed when the cervical lymph nodes were deemed to be potentially positive based on the preoperative assessment. Retrosternal or posterior mediastinal routes were performed for esophageal reconstructions with gastric conduits or colons. All resected specimens were labeled by the surgeon and submitted for pathologic examination. Lymph node involvement was determined based on the 7thAJCC/TNM staging system[9].

\section{Fibrinogen and Albumin Measurements}

The levels of preoperative fibrinogen and albumin were examined from blood samples obtained before breakfast within two weeks before surgery. The samples were promptly centrifuged and processed within two hours. The fibrinogen was processed by a CA-7000 automatic coagulation analyzer (Sysmex Corporation, Kobe, Japan). The levels of fibrinogen were measured by reagents (Siemens AG, Munich, Germany) provided through a Diagon Dia-Timer 4 (Diagon Ltd, Budapest, Hungary). We used the bromocresol green(BCG) dye method to measure the levels of the serum albumin.
The FAR was defined as the fibrinogen levels divided by the serum albumin levels. The GPS and modified GPS (mGPS) were defined as before[10,11].

\section{Follow up}

Followed up in our center was recommended every three months during the first two years, then every six months for the next three years and annually afterwards. The protocol includes medical history and physical examination, chest radiography, esophageal radiography, and/or CT from the neck to the upper abdomen. Upper endoscopy or PET were performed as clinically indicated recurrence or metastasis. January 2016 was the last follow up date.

\section{Statistical Analysis}

We used SPSS 17.0 software (SPSS Inc., Chicago, IL, USA) to perform the statistical analysis. The association between the FAR and clinicopathologic factors was assessed by the Chi-square test. The correlation between FAR and CRP, and NLR were assessed through linear correlation analysis. The survival time was assessed by the Kaplan-Meier method. The difference in survival between groups were estimated by the log-rank test. Multivariate analyses were used to evaluate the independent prognostic factors for OS by the Cox regression proportional hazard model. A $p$-value less than 0.05 was considered statistically significant. All cut points were assessed by the X-tile software (http://www.tissuearray.org/rimmlab/)[12], identifying the cutoff with the minimum $p$-value according to the survival status.

\section{Results}

We enrolled 1135 consecutive ESCC patients in our study, including 888 (78.2\%) males and 247 $(21.8 \%)$ females. The median age of the patients was 58 years (range, 28-88). The clinicopathological features of patients are shown in Table 1 . The median FAR was 0.078 (range, $0.01-0.3$ ). Using X-tile software, the cutoff of FAR is 0.08 in our study. We divided patients into two groups: the low $(<0.08)$ FAR group $(\mathrm{n}=625)$ and high $(\geq 0.08)$ FAR group $(\mathrm{n}=510)$.

The FAR was statistically significantly associated with age $(p=0.003)$, $\operatorname{sex}(p=0.030)$, tumor length $(p=0.043), \quad \mathrm{pT}$ status $(p<0.001)$ and $\mathrm{pN}$ status $(p<0.001)$, whereas the FAR was not statistically significantly associated with tumor location $(p=0.561)$, and tumor grade $(p=0.096)$. Pearson's correlation indicated that the FAR levels were positively associated with the serum CRP levels $(r=0.583, p$ $<0.001$ ) (Fig. 1a). The FAR levels were also positively associated with the NLR levels $(r=0.316, p<0.001)$ (Fig. $1 b)$. 
Table 1. Correlation of the FAR with the clinicopathological features of patients.

\begin{tabular}{|c|c|c|c|}
\hline Variables & Patients & & $p$-value \\
\hline & FAR $<0.08$ & FAR $\geq 0.08$ & \\
\hline Sex & & & 0.030 \\
\hline male & 474 & 414 & \\
\hline female & 151 & 96 & \\
\hline Age & & & 0.003 \\
\hline$>60$ & 404 & 286 & \\
\hline$\leq 60$ & 221 & 224 & \\
\hline Tumor location & & & 0.561 \\
\hline Upper & 151 & 126 & \\
\hline Middle & 311 & 265 & \\
\hline Lower & 163 & 119 & \\
\hline Tumor length & & & 0.043 \\
\hline$\leq 3 \mathrm{~cm}$ & 349 & 254 & \\
\hline$>3 \mathrm{~cm}$ & 276 & 256 & \\
\hline Tumor grade & & & 0.096 \\
\hline good & 131 & 132 & \\
\hline moderate & 312 & 227 & \\
\hline poor & 182 & 151 & \\
\hline pT status & & & $<0.001$ \\
\hline $\mathrm{T} 1$ & 124 & 49 & \\
\hline $\mathrm{T} 2$ & 131 & 82 & \\
\hline $\mathrm{T} 3$ & 368 & 373 & \\
\hline $\mathrm{T} 4 \mathrm{a}$ & 2 & 6 & \\
\hline pN status & & & 0.005 \\
\hline N0 & 344 & 229 & \\
\hline N1 & 264 & 257 & \\
\hline N2 & 16 & 22 & \\
\hline N3 & 1 & 2 & \\
\hline
\end{tabular}

The five-year survival rate in our cohort was $49.8 \%$. The survival rate decreased in high FAR patients compared with low FAR patients $(p<0.001$; Fig. 2). Univariate analysis indicated that age, tumor grade, pT status, $\mathrm{pN}$ status, GPS, mGPS, NLR, PLR, LMR and FAR were prognostic factors. Multivariate analysis indicated that age, tumor grade, pT status, $\mathrm{pN}$ status and FAR were independent prognostic factors in patients with ESCC (Table 2).

Table 2. Univariate and multivariate analysis in overall survival

\begin{tabular}{lllll}
\hline \multicolumn{4}{l}{ Variables } & \multicolumn{3}{l}{ Univariate Analysis } & \multicolumn{2}{l}{ Multivariate Analysis } \\
\hline & $p$-Value & HR & $95 \%$ CI & $p$-Value \\
\hline sex & 0.608 & & & \\
Age & $<0.001$ & 1.491 & $(1.229-1.809)$ & $<0.001$ \\
Tumor location & 0.134 & & & \\
Tumor length & 0.128 & & & \\
Tumor grade & 0.004 & 1.192 & $(1.039-1.368)$ & 0.012 \\
pT status & $<0.001$ & 1.288 & $(1.109-1.496)$ & $<0.001$ \\
pN status & $<0.001$ & 1.538 & $(1.378-1.717)$ & $<0.001$ \\
GPS & $<0.001$ & 2.081 & $(0.832-5.203)$ & 0.117 \\
mGPS & $<0.001$ & 0.457 & $(0.180-1.159)$ & 0.099 \\
NLR & $<0.001$ & 1.267 & $(0.989-1.624)$ & 0.061 \\
PLR & 0.002 & 1.088 & $(0.853-1.389)$ & 0.496 \\
MLR & 0.001 & 0.995 & $(0.804-1.231)$ & 0.965 \\
FAR & $<0.001$ & 1.325 & $(1.087-1.691)$ & 0.012 \\
Fibrinogen & $<0.001$ & 1.189 & $(0.879-1.608)$ & 0.261 \\
Albumin & $<0.001$ & 0.818 & $(0.637-1.056)$ & 0.123 \\
\hline
\end{tabular}
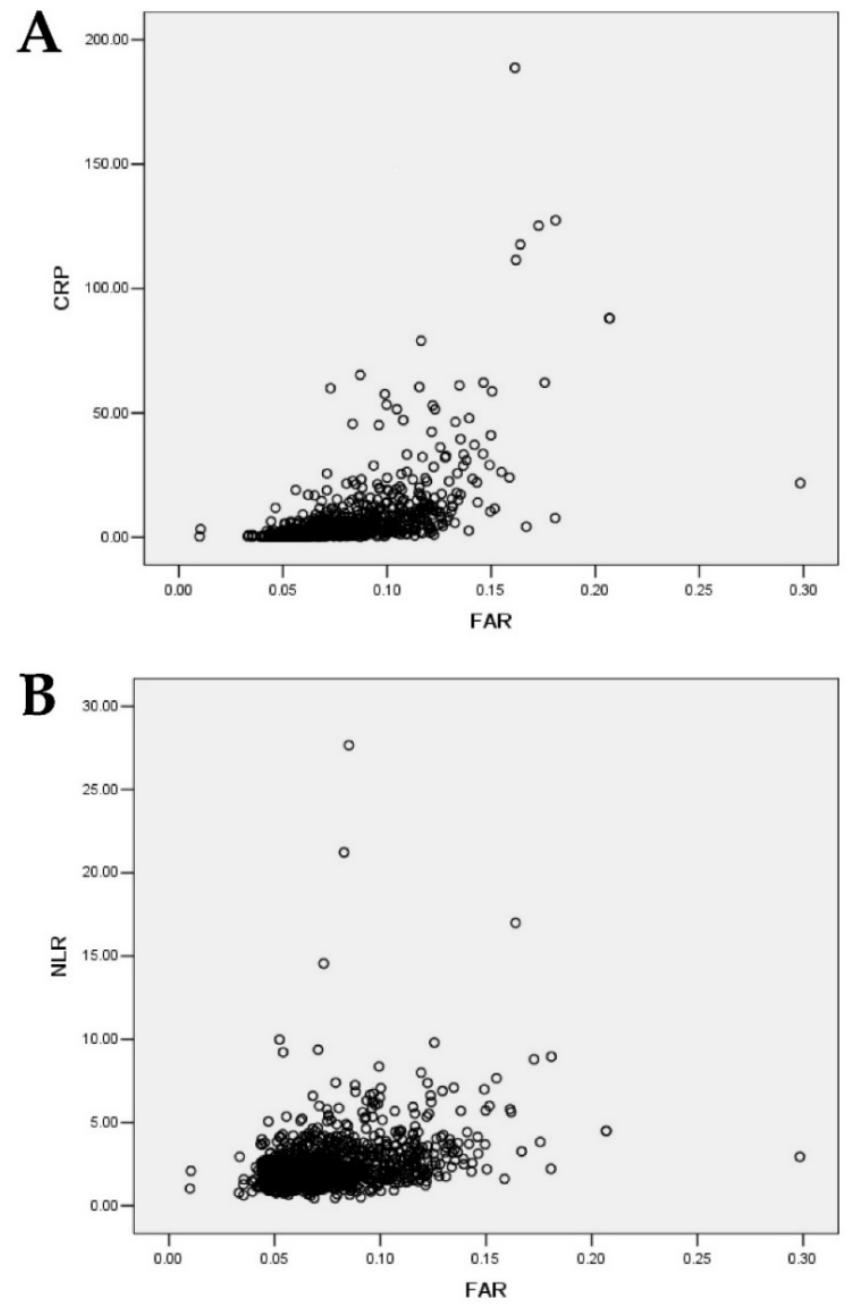

Figure 1. (a) Correlations between FAR and $\operatorname{CRP}(r=0.583, p<0.001)$. (b) Correlations between FAR and NLR $(r=0.316, p<0.001)$.

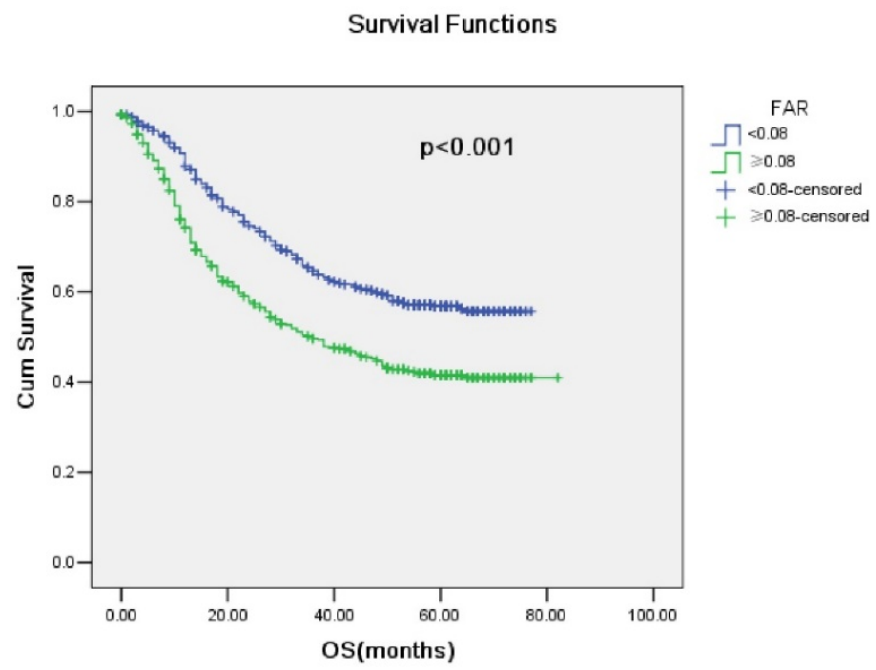

Figure 2. Kaplan-Meier survival curves stratified by $\operatorname{FAR}(p<0.001)$ 


\section{Discussion}

Investigators have demonstrated that plasma fibrinogen and serum albumin are thought to be associated with poor outcomes in ESCC patients $[8,13]$. Therefore, we proposed a novel prognostic indicator based on a combined analysis of fibrinogen and albumin in patients with ESCC. In our study, higher FAR was thought to be associated with a number of important clinicopathological parameters shown to be predictive of worse outcomes. Moreover, higher FAR was also associated with poor survival in ESCC patients, which indicated that elevated FAR might be associated with aggressive burden and systemic progression of ESCC.

Previous reports have indicated that the hypercoagulable state is thought to be related to a more malignant disease $[5,6,13]$. However, the mechanism underlying the relationship between the level of fibrinogen and cancer progression remains unclear. Several potential mechanisms may explain the prognostic values of the level of fibrinogen in cancer. Fibrinogen acts like a dimeric molecular bridge to promote cell to cell adhesion. Higher fibrinogen receptors, integrin or non-integrin (eg, intercellular adhesion molecule 1), are usually expressed by malignant cells. Fibrinogen connects malignant cells and vascular endothelium to enhance tumor progression and metastasis[14,15]. Furthermore, platelets also have a fibrinogen receptor (eg, aII b3 integrin). Fibrinogen promotes the bridge between platelets and malignant cells, which can be easier to form microemboli in targeted organs, increasing in parallel with tumor progression and metastasis[16]. Moreover, fibrinogen may produce signals to tie up growth factors, such as vascular endothelial cell and fibroblast growth factors, promoting malignant cell proliferation and angiogenesis[17]. In addition, several studies reported that anticoagulants, such as low-molecular-weight heparins and heparins, may prolong survival in cancer patients by preventing the progression of metastasis[18,19]. Although further investigations are essential to validate the effectiveness and safety of anticoagulants, it would seem that anticoagulation therapy may help to prolong survival in cancer patients.

Fibrinogen is not only an essential component of the coagulation cascade but also an acute-phase reactant reflecting a state of systemic inflammation. It has been reported that fibrinogen plays an important role on the process of inflammation, promoting proinflammatory cytokines synthesis in peripheral blood inflammatory environment [20,21]. An interesting animal experiment showed that fibrinogen might be a critical factor of the metastatic potential in lung carcinoma and melanoma cells. The development of metastasis was decreased in mice lacking fibrinogen $[22,23]$. The findings set up a significant connection between coagulation and natural immune system. It may demonstrate that the fibrinogen-platelet link administers to metastatic development by helping malignant cells to prevent natural killer cell with thrombin. Fibrinogen-platelet microthrombi may provide a defense to keep malignant cells from the natural immunity and avoid removal of malignant cells [22]. Additionally, it is proven that interleukin-6 (IL-6), an inflammatory protein, has been found to be a critical regulator of hyperfibrinogenemia [24]. Therefore, hyperfibrinogenemia is deemed to be related to the overproduction of inflammatory cytokines such as IL-6 in malignant cells, suggesting that inflammatory status and tumor aggressiveness behavior might be reflected by fibrinogen levels.

Albumin is an indicator in the assessment of nutritional status. Several studies have reported that lower serum albumin may result in a deterioration of disease and a high risk of poor outcome in patients with cancer $[8,25]$. It is demonstrated that malnutrition may weaken the immune system, increasing the chance of infection and further accelerating the progression of malignancy [8]. Furthermore, a low serum albumin level is also a marker of systemic inflammatory response, promoting tumor proliferation, invasion, and migration. It is found that albumin may aid to stabilize DNA replication and cell growth, regulate body reaction, strength natural immunity and prevent malignant disease [25].

FAR, which absorbs the fibrinogen and albumin, suggests that it might combine the predictive effects of those indexes and reflect a mixed prognostic value. In our study, we found a positive correlation between FAR and the inflammatory indicator CRP or NLR. Thus, FAR was related with not only a coagulation and nutrition based index, but also a inflammation based index. For all we know, this is the first study concentrating on the prognostic impact of FAR in ESCC. Both fibrinogen and albumin are routinely measured in clinical practice. The measurements are inexpensive, convenient and repeatable. Furthermore, the present study also found that FAR correlated with longer tumor length, deeper tumor invasion, and increased burden of the regional lymph node status in ESCC patients. It indicated that FAR might help to identify a subgroup of patients who were considered to be borderline candidates based on conventional imaging techniques. It may assist in the selection of patients with ESCC for neoadjuvant chemoradiotherapy in preoperative management. 
However, our study was limit to its retrospective design including the data collection. Moreover, as the data were from a single center, whether our findings can be applicable to other centers needs to be validated. Furthermore, although the current guidelines recommended that induction chemoradiation followed by surgery is the optimal treatment strategy for locally advanced esophageal cancer patients, neoadjuvant chemoradiation were not commonly undertaken before 2010 in our center. Therefore, larger sample sizes are needed for verification.

In conclusion, our present study indicates that preoperative FAR was an independent prognostic factor in ESCC patients. Lower FAR may improve OS of ESCC patients.

\section{Acknowledgment}

We thank the members of Guangdong Esophageal Cancer Institute for their cooperation and assistance.

This work was supported by National Natural Science Foundation of China (No. 81402003), Guangzhou Science and Technology Program (2014Y2-00143), Sun Yat-sen University medical young teacher training program (15ykpy34), and Sun Yat-sen University Clinical Research 5010 Program (No. 2007048).

\section{Competing Interests}

The authors have declared that no competing interest exists.

\section{References}

1. Jemal A, Bray F, Center MM, et al. Global cancer statistics. CA Cancer J Clin.2011; 61:69-90.

2. Lin Y, Totsuka Y, He Y, Kikuchi S, Qiao Y, Ueda J, et al. Epidemiology of esophageal cancer in Japan and China. J Epidemiol. 2013;23(4):233-42.

3. Hagen P, Hulshof MC, van Lanschot JJ, et al. Preoperative chemoradiotherapy for esophageal or junctional cancer. N Engl J Med. 2012 ;366(22):2074-84.

4. Mariette C, Dahan L, Mornex F, et al. Surgery Alone Versus Chemoradiotherapy Followed by Surgery for Stage I and II Esophageal Cancer: Final Analysis of Randomized Controlled Phase III Trial FFCD 9901. J Clin Oncol.2014;32(23):2416-22.

5. Ghanim B, Hoda MA, Klikovits T, et al. Circulating fibrinogen is a prognostic and predictive biomarker in malignant pleural mesothelioma. Br J Cancer. 2014;110(4):984-90.

6. Son HJ, Park JW, Chang HJ, et al. Preoperative Plasma Hyperfibrinogenemia is Predictive of Poor Prognosis in Patients with Nonmetastatic Colon Cancer. Ann Surg Oncol .2013;20(9):2908-2913

7. Weisel JW. Fibrinogen and fibrin. Adv Protein Chem. 2005;70:247-299.

8. Gupta D, Lis CG. Pretreatment serum albumin as a predictor of cancer survival: a systematic review of the epidemiological literature. Nutr J. 2010 ;9:69

9. Edge SB, Compton CC. The American Joint Committee on Cancer: the 7th edition of the AJCC cancer staging manual and the future of TNM. Ann Surg Oncol.2010; 17: 1471-4.

10. Crumley AB, McMillan DC, McKernan $M$, et al. Evaluation of an inflammation-based prognostic score in patients with inoperable gastro-oesophageal cancer. Br J Cancer.2006;94:637-641.

11. Proctor MJ, Morrison DS, Talwar D, et al. An inflammation-based prognostic score (mGPS) predicts cancer survival independent of tumour site: a Glasgow Inflammation Outcome Study. Br J Cancer.2011;104:726-734.

12. Camp RL, Dolled FM, Rimm DL. X-tile: a new bio-informatics tool for biomarker assessment and outcome-based cut-point optimization. Clin Cancer Res.2004; 10: 7252-9.
13. Takeuchi H, Ikeuchi S, Kitagawa Y, et al. Pretreatment plasma fibrinogen level correlates with tumor progression and metastasis in patients with squamous cell carcinoma of the esophagus. J Gastroenterol Hepatol. 2007;22:2222-7.

14. Steinbrecher KA, Horowitz NA, Blevins EA, et al. Colitis-associated cancer is dependent on the interplay between the hemostatic and inflammatory systems and supported by integrin alpha(M)beta(2) engagement of fibrinogen. Cancer Res. 2010;70:2634-43.

15. Yano HKitayama J, Hatano K, Tsuno N, et al. Clustered cancer cells show a distinct adhesion behavior from single cell form under physiological shear conditions. J Exp Clin Cancer Res. 2001;20:407-12.

16. Zheng S, Shen J, Jiao $Y$, et al. Platelets and fibrinogen facilitate each other in protecting tumor cells from natural killer cytotoxicity. Cancer Sci. 2009;100:859-65.

17. Simpson-Haidaris PJ, Rybarczyk B. Tumors and fibrinogen. The role of fibrinogen as an extracellular matrix protein. Ann $N$ Y Acad Sci. 2001;936:406-25.

18. Bobek V, Kova r J. Antitumor and antimetastatic effect of warfarin and heparins . Biomed Pharmac other 2004; 58: 213-9.

19. Kuderer NM, Khorana AA, Lyman GH, et al. A meta-analysis and systematic review of the efficacy and safety of anticoagulants as cancer treatment: impact on survival and bleeding complications. Cancer 2007; 110: 1149-61.

20. Jensen T, Kierulf P, Sandset PM, et al. Fibrinogen and fibrin induce synthesis of proinflammatory cytokines from isolated peripheral blood mononuclear cells. Thromb Haemost 2007;97:822- 829.

21. Yakovlev S, Zhang L, Ugarova $T$, et al. Interaction of fibrin(ogen) with leukocyte receptor alpha $\mathrm{M}$ beta 2 (Mac-1): Further characterization and identification of a novel binding region within the central domain of the fibrinogen gamma-module. Biochemistry 2005;44:617- 626 .

22. Palumbo JS, Talmage KE, Massari JV et al. Platelets and fibrin(ogen) increase metastatic potential by impeding natural killer cell-mediated elimination of tumor cells. Blood 2005;105:178 -185.

23. Palumbo JS, Kombrinck KW, Drew AF et al. Fibrinogen is an important determinant of the metastatic potential of circulating tumor cells. Blood 2000;96:3302-3309.

24. Albrecht U, Yang X, Asselta R, et al. Activation of NF-kappa B by IL-1 beta blocks IL-6-induced sustained STAT3 activation and STAT3-dependent gene expression of the human gamma-fibrinogen gene. Cell Signal. 2007;19(9):1866-78

25. Lis CG, Gupta D, Lammersfeld CA, Markman M, Vashi PG. Role of nutritional status in predicting quality of life outcomes in cancer-a systematic review of the epidemiological literature. Nutr J. 2012;11:27. 